\title{
EFFICIENT NUMERICAL METHODS FOR SIMULATING SURFACE TENSION OF MULTI-COMPONENT MIXTURES WITH THE GRADIENT THEORY OF FLUID INTERFACES *
}

\author{
JISHENG KOU ${ }^{\dagger}$, SHUYU SUN ${ }^{\ddagger}$, AND XIUHUA WANG ${ }^{\S}$
}

\begin{abstract}
Surface tension significantly impacts subsurface flow and transport, and it is the main cause of capillary effect, a major immiscible two-phase flow mechanism for systems with a strong wettability preference. In this paper, we consider the numerical simulation of the surface tension of multi-component mixtures with the gradient theory of fluid interfaces. Major numerical challenges include that the system of the Euler-Lagrange equations is solved on the infinite interval and the coefficient matrix is not positive definite. We construct a linear transformation to reduce the Euler-Lagrange equations, and naturally introduce a path function, which is proven to be a monotonic function of the spatial coordinate variable. By using the linear transformation and the path function, we overcome the above difficulties and develop the efficient methods for calculating the interface and its interior compositions. Moreover, the computation of the surface tension is also simplified. The proposed methods do not need to solve the differential equation system, and they are easy to be implemented in practical applications. Numerical examples are tested to verify the efficiency of the proposed methods.
\end{abstract}

Key words. Surface tension; Multi-component mixtures; Euler-Lagrange equations; Gradient theory

AMS subject classifications. 65N30; 65N50; 49S05

1. Introduction. Subsurface flow and transport often involve multiple fluid phases; that is, subsurface phenomena often come with mixture of immiscible and partially miscible fluids. Subsurface oil and gas reservoirs typically contain oil phase, gas phase and water phase, together with the solid phase (rock or soil) [10]. Modeling and simulation of multiphase fluid systems have been a major effort in reservoir engineering [16]. In addition to oil reservoir management, understanding and modeling of multiphase systems are also crucial to many environment issues $[29,30]$. For example, one of the most attractive and practical solutions to reduce the $\mathrm{CO}_{2}$ emission problem is to inject and store $\mathrm{CO}_{2}$ in the subsurface geological formations [21], such as depleted reservoirs and deep saline aquifers. The large capacity of subsurface storage provides several advantages over other possible alternatives of carbon sequestration. For subsurface carbon sequestration, there are four well-accepted major mechanisms to trap the injected $\mathrm{CO}_{2}$ for long-term storage, namely, structural (stratigraphic) trapping, residual fluid trapping, solubility trapping and mineral trapping; all of the four major mechanisms are directly or indirectly related to phase behaviors of fluid systems.

At a pore scale, a surface or an interface exists between any two immiscible or partially miscible fluids and between a fluid and a solid. It is well known that at the

*This work is supported by National Natural Science Foundation of China (No.11301163), the Key Project of Chinese Ministry of Education (No.212109) and the KAUST faculty research fund.

${ }^{\dagger}$ School of Mathematics and Statistics, Hubei Engineering University, Xiaogan 432000, Hubei, China.

${ }^{\ddagger}$ Corresponding author. Computational Transport Phenomena Laboratory, Division of Physical Science and Engineering, King Abdullah University of Science and Technology, Thuwal 23955-6900, Kingdom of Saudi Arabia; School of Mathematics and Statistics, Xi'an Jiaotong University, Xi'an 710049, China. Email: shuyu.sun@kaust.edu.sa.

$\S$ School of Mathematics and Statistics, Hubei Engineering University, Xiaogan 432000, Hubei, China. 
molecular level of a liquid and gas interface for instance, the interior molecules of the liquid phase experience zero (or negligible) net attractive forces from the surrounding molecules because the molecules are all similar with approximately equal spacing and equal attraction in all directions. However, on the interface, the molecules experience a stronger attractive pull towards the interior of the liquid body, since fluid molecules do not surround the interface molecule equally in all directions. This anisotropic attractive force yields interfacial tension and impacts the shape of the liquid-gas interface. At the Darcy scale, we do not model the liquid-gas interfaces explictly [27, 28], but the existence of these liquid-gas interfaces manifests themselves by many Darcy-scale phenomena.

Capillary effect (or capillarity) is perhaps one of the most important Darcy-scale phenomena arising from the pore-scale liquid-gas interfaces. Capillary effect and its resultant capillary pressure are caused by surface tension between immiscible (or partially miscible) fluids, and it is one of major forces in fluid (oil, gas and water) flow and transport in subsurface [16]. In fact, the capillary effect is frequently the leading mechanism of oil recovery in fractured oil reservoirs. In addition to capillary pressure, surface tension also significantly influences other important parameters of porous medium processes including relative permeability and residual saturations, thus substantially impacts the flow and the transport of the vapour and liquid phases in a porous medium; consequently, it plays important roles in a number of chemical and reservoir engineering problems $[3,19,20]$.

To simulate the subsurface multiphase flow and transport accurately and efficiently, it is important to model and compute the surface tension of the multiphase mixture properly. There are a few models in the literature for simulating the multicomponent surface, for example, the gradient theory of fluid interfaces [8] and inhomogeneous molecular modeling simulation of multicomponent surface [17, 22, 32]. In this paper, we focus on the gradient theory of fluid interfaces, which has been a frequently-used method to model and predict the surface tension $[3,8,19,20]$. In our previous work [15], we have developed an efficient computational scheme to model and calculate the surface tension of single-species two-phase fluid systems based on our rigorous mathematical re-formulation of the gradient theory. In the work presented in this paper, we propose a few efficient computational methods to calculate the surface tension of multiple-species two-phase fluid systems. We note that the work presented here is not a simple extension of our previous work; instead, new computational treatment is proposed to address the challenges of multiple-species systems.

In the gradient theory, the Helmholtz energy density of an inhomogeneous fluid, in the absence of an external potential, is the sum of two contributions: the Helmholtz energy of homogeneous fluid at local composition, and a corrective term, which is function of the local density gradients. By applying the minimum free energy criterion, the compositions in the interface must satisfy the following Euler-Lagrange equations $[15,19,20]$

$$
\sum_{j=1}^{N} c_{i j} \frac{d^{2} n_{j}}{d x^{2}}=\mu_{i}^{0}-\mu_{i}, \quad i=1, \cdots, N
$$

where $N$ is the component number of a mixture, $\mathbf{n}=\left[n_{1}, n_{2}, \cdots, n_{N}\right]^{T}$ denotes the molar densities, and the coefficients $c_{i j}$ denote the influence parameters. To describe the definitions of $\mu_{i}^{0}$ and $\mu_{i}$, we first need to define the Helmholtz energy of homogeneous fluid by $f_{0}(\mathbf{n})$, and its expression is given in Appendix A. From this, the 
chemical potential of component $i$ is defined by

$$
\mu_{i}^{0}=\left(\frac{\partial f_{0}(\mathbf{n} ; T)}{\partial n_{i}}\right)_{T, n_{1}, \cdots, n_{i-1}, n_{i+1}, \cdots, n_{N}},
$$

where $T$ is the temperature. In addition, $\mu_{i}$ is the chemical potential in the equilibrium bulk phases.

Let $c_{i}$ be the pure component influence parameters. The computation of $c_{i}$ is described in Appendix B. The crossed influence parameters $c_{i j}$ are expressed as the modified geometric mean of the pure component influence parameters $c_{i}$ and $c_{j}$ by

$$
c_{i j}=\left(1-\beta_{i j}\right) \sqrt{c_{i} c_{j}},
$$

where the parameters $\beta_{i j}$ are binary interaction coefficients for the influence parameters. Stability of the interface requires $\beta_{i j}$ to be included between 0 and 1 ; if taking $\beta_{i j}=0$, the mixing rule becomes the simple geometric mean. It has been shown in $[19,20]$ that the different choices of $\beta_{i j}$ have little effect on the surface tension computation. The usual choice strategy is to take $\beta_{i j}=0$, and thus, we pay attention to the case $\beta_{i j}=0$ in this paper. We assume that the influence parameters are independent of spatial positions and the dependence of the influence parameters on density is also neglected $[19,20]$.

The Euler-Lagrange equations is closed with the boundary condition, which is given by

$$
\mathbf{n} \rightarrow \mathbf{n}^{G} \text {, as } x \rightarrow-\infty \text {, and } \mathbf{n} \rightarrow \mathbf{n}^{L} \text {, as } x \rightarrow+\infty .
$$

Here, $\mathbf{n}^{G}$ and $\mathbf{n}^{L}$ denote the mixture compositions of the equilibrium bulk gas and liquid phases, respectively, which can be calculated by the Peng-Robinson equation of state. We assume that there exists a sufficiently smooth solution $\mathbf{n}$ satisfying the Euler-Lagrange equations (1.1) with the boundary condition (1.4).

By solving the Euler-Lagrange equations, we calculate the molar densities of components at the liquid-gas interface, and based on the density distributions, we can further calculate the magnitude of surface tension at the interface $[19,20]$. The surface tension, denoted by $\sigma$, is expressed as

$$
\sigma=\int_{-\infty}^{+\infty}\left(\Phi(\mathbf{n})-\Phi_{B}+\sum_{i, j=1}^{N} \frac{1}{2} c_{i j} \frac{d n_{i}}{d x} \frac{d n_{j}}{d x}\right) d x,
$$

where $\Phi(\mathbf{n})$ is the grand thermodynamic potential defined as

$$
\Phi(\mathbf{n})=f_{0}(\mathbf{n})-\sum_{i=1}^{N} \mu_{i} n_{i},
$$

and $\Phi_{B}$ is the value of $\Phi(\mathbf{n})$ in the equilibrium bulk phases. In (1.6), $\mu_{i}$ is the chemical potential of component $i$ in the equilibrium bulk phases; that is, $\mu_{i}=\mu_{i}^{0}\left(\mathbf{n}^{G}\right)=$ $\mu_{i}^{0}\left(\mathbf{n}^{L}\right)$.

In the surface tension computation stated above, there exist two key problems, which are different from the classical phase-field models $[1,2,4,6,9,11-13,18,23,25,31]$ and the gradient theory model [15]. The first problem is to solve a boundary value problem on the infinite interval $(-\infty,+\infty)$. Moreover, the computation of the surface 
tension mainly depends on the compositions within the two-phase interface, so it is necessary to design the efficient methods for computing both the interface and its interior compositions. The key difficulty is to accurately capture the interface between two phases. The second problem is that the influence parameter matrix, denoted by $\mathbf{C}=\left(c_{i j}\right)_{i, j=1}^{N}$, is not positive definite, and thus, the Euler-Lagrange equations can not be solved by the standard approaches.

In order to resolve these two problems, the path function methods have been developed in the references $[5,7,26]$. The most popular one is the method of selecting the reference component as the path function $[3,8,19,20]$. By the selection of the reference component, the Euler-Lagrange equations can be reduced with the transformations to a solvable system of nonlinear equations, and then the expressions of the surface tension and density distributions are derived. Its principal difficulty is the selection of the reference component, which requires the monotonicity of the reference component in the two-phase interface. The selection strategy is usually based on the experiences, but without any mathematical justification.

In this paper, we will firstly construct a linear transformation to reduce the EulerLagrange equations such that they are convenient to be solved, and naturally derive a path function, which is different from the reference component. This path function is similar to the one mentioned in [7], but which is not used since its monotonicity has never been established. Based on the new formulation of this path function, we will prove its monotonicity, which allows us not only to develop two efficient methods for calculating the two-phase interface, but also to reduce the computation of the surface tension. From this, we will propose the efficient and accurate methods for computing the surface tension, along with the interface and its interior compositions. Compared to the known methods, the proposed methods are easier to be implemented in practical applications. Three numerical examples are also given to verify the efficiency of the proposed methods.

\section{Reduced mathematical model.}

2.1. Linear transformation. Let $\mathbf{c}=\left[\sqrt{c_{1}}, \cdots, \sqrt{c_{N}}\right]^{T}$, then the influence matrix is expressed as $\mathbf{C}=\left(c_{i j}\right)_{i, j=1}^{N}=\mathbf{c c}^{T}$. It is easy to find that $\mathbf{C}$ possesses a unique nonzero eigenvalue, denoted by $\lambda$, i.e.

$$
\lambda=\mathbf{c}^{T} \mathbf{c}=\sum_{i=1}^{N} c_{i},
$$

and its corresponding eigenvector

$$
\mathbf{v}_{1}=\frac{\mathbf{c}}{\sqrt{\lambda}}=\frac{1}{\sqrt{\sum_{i=1}^{N} c_{i}}}\left[\sqrt{c_{1}}, \cdots, \sqrt{c_{N}}\right]^{T} .
$$

As $\mathbf{C}$ is a symmetrical matrix, there exist $N-1$ zero eigenvalues of $\mathbf{C}$, and the corresponding eigenvectors, denoted by $\mathbf{v}_{i}, 2 \leq i \leq N$, such that

$$
\mathbf{C v}_{i}=0, \quad 2 \leq i \leq N .
$$

The vector set $\left\{\mathbf{v}_{i}\right\}_{i=2}^{N}$ is a basis of the null subspace of $\mathbf{v}_{1}$; that is, $\mathbf{v}_{i} \in \mathcal{N}\left(\mathbf{v}_{1}\right)=$ $\left\{\mathbf{v} \in \mathbb{R}^{N}: \mathbf{v}^{T} \mathbf{v}_{1}=0\right\}$. One choice strategy of $\mathbf{v}_{i}$ is to take

$$
\mathbf{v}_{i}(1)=\frac{\sqrt{c_{i}}}{\sqrt{\lambda}}, \quad \mathbf{v}_{i}(i)=-\frac{\sqrt{c_{1}}}{\sqrt{\lambda}}, \quad 2 \leq i \leq N,
$$




$$
\mathbf{v}_{i}(j)=0, \quad 2 \leq i \leq N, \quad 2 \leq j \leq N, \quad j \neq i .
$$

It is easy to check $\mathbf{C v}_{i}=\mathbf{c c}^{T} \mathbf{v}_{i}=0$ for $2 \leq i \leq N$. By a simple deduction, we find that $\mathbf{v}_{i} \in \mathcal{N}\left(\mathbf{v}_{1}\right), 2 \leq i \leq N$, and the vector group $\left\{\mathbf{v}_{i}\right\}_{i=1}^{N}$ is linearly independent.

Define a transformation matrix $\mathbf{Q}=\left[\mathbf{v}_{1}, \cdots, \mathbf{v}_{N}\right]$. It follows from the definitions of $\mathbf{v}_{i}$ that $\mathbf{Q}$ is invertible. From this, we define a vector $\mathbf{u}=\left[u, u_{2}, \cdots, u_{N}\right]^{T}$ as

$$
\mathbf{u}=\mathbf{Q}^{-1} \mathbf{n}, \quad \mathbf{n}=\mathbf{Q u} .
$$

With simple calculation, it is obtained from (2.2) that

$$
u=\mathbf{v}_{1}^{T} \mathbf{n}=\frac{1}{\sqrt{\lambda}} \sum_{i=1}^{N} \sqrt{c_{i}} n_{i} .
$$

Here, we call $u$ as the weighted molar density of a mixture, and $u$ will be used as the path function. Note that $\mathbf{n}$ and $\mathbf{u}$ are the vector functions varying with the spatial location, but $\mathbf{Q}$ is independent of spatial positions. The following relations are obtained from the definitions:

$$
\begin{gathered}
\frac{\partial n_{1}}{\partial u}=\frac{\sqrt{c_{1}}}{\sqrt{\lambda}}, \quad \frac{\partial n_{1}}{\partial u_{i}}=\frac{\sqrt{c_{i}}}{\sqrt{\lambda}}, \quad 2 \leq i \leq N, \\
\frac{\partial n_{i}}{\partial u}=\frac{\sqrt{c_{i}}}{\sqrt{\lambda}}, \quad \frac{\partial n_{i}}{\partial u_{i}}=-\frac{\sqrt{c_{1}}}{\sqrt{\lambda}}, \quad 2 \leq i \leq N, \\
\frac{\partial n_{i}}{\partial u_{j}}=0, \quad 2 \leq i, j \leq N, \quad i \neq j .
\end{gathered}
$$

2.2. Reduced Euler-Lagrange equations. By the above linear transformation, we now reduce the Euler-Lagrange equations (1.1). Denote $\boldsymbol{\mu}=\left[\mu_{1}, \cdots, \mu_{N}\right]^{T}$, $\boldsymbol{\mu}^{0}=\left[\mu_{1}^{0}, \cdots, \mu_{N}^{0}\right]^{T}$ and $\delta \boldsymbol{\mu}=\boldsymbol{\mu}^{0}-\boldsymbol{\mu}$. The matrix-vector form of (1.1) is expressed as

$$
\mathbf{C} \frac{d^{2} \mathbf{n}}{d x^{2}}=\delta \boldsymbol{\mu}
$$

Multiplying the equation (2.7) by $\mathbf{v}_{1}$, we find that the left-hand side of (2.7) becomes

$$
\mathbf{v}_{1}^{T} \mathbf{C} \frac{d^{2} \mathbf{n}}{d x^{2}}=\mathbf{v}_{1}^{T} \mathbf{C Q} \frac{d^{2} \mathbf{u}}{d x^{2}}=[\lambda, 0, \cdots, 0] \frac{d^{2} \mathbf{u}}{d x^{2}}=\lambda \frac{d^{2} u}{d x^{2}} .
$$

As a result, we obtain the first equation as

$$
\lambda \frac{d^{2} u}{d x^{2}}=\mathbf{v}_{1}^{T} \delta \boldsymbol{\mu} .
$$

Similarly, the property of $\mathbf{v}_{i}$ gives us

$$
\mathbf{v}_{i}^{T} \mathbf{C} \frac{d^{2} \mathbf{n}}{d x^{2}}=0, \quad 2 \leq i \leq N,
$$

which yields

$$
\mathbf{v}_{i}^{T} \delta \boldsymbol{\mu}=-\lambda^{-1 / 2}\left(\sqrt{c_{1}} \delta \mu_{i}-\sqrt{c_{i}} \delta \mu_{1}\right)=0, \quad 2 \leq i \leq N .
$$


Thus, the Euler-Lagrange equations can be reduced as

$$
\begin{gathered}
\lambda \frac{d^{2} u}{d x^{2}}=\sum_{i=1}^{N} \sqrt{c_{i} / \lambda}\left(\mu_{i}^{0}-\mu_{i}\right), \\
\sqrt{c_{1}}\left(\mu_{i}^{0}-\mu_{i}\right)=\sqrt{c_{i}}\left(\mu_{1}^{0}-\mu_{1}\right), \quad 2 \leq i \leq N .
\end{gathered}
$$

It is also obtained from (2.12) and (2.13) that

$$
\begin{gathered}
\sqrt{\lambda c_{1}} \frac{d^{2} u}{d x^{2}}=\mu_{1}^{0}-\mu_{1}, \\
\sqrt{c_{j}}\left(\mu_{i}^{0}-\mu_{i}\right)=\sqrt{c_{i}}\left(\mu_{j}^{0}-\mu_{j}\right), \quad 1 \leq i, j \leq N .
\end{gathered}
$$

2.3. Monotonicity of $u(x)$. To select $u$ as the path function, its monotonicity is a necessary condition, so we now prove this key property. The composition in each bulk phase region is homogenous, i.e., $\frac{d n_{i}}{d x}=0$ and thus, $\frac{d u}{d x}=0$ within the bulk phase regions. The Euler-Lagrange equations given by (2.12) and (2.13) hold not only in the interface region but also in the bulk phase regions. The above two facts allow us to determine the interface and its interior compositions. Then we know that as $x \rightarrow \pm \infty, \frac{d n_{i}}{d x} \rightarrow 0$ and thus, $\frac{d u}{d x} \rightarrow 0$. Multiplying (2.12) by $\frac{d u}{d x}$ and integrating it over $(-\infty, x)$, we get

$$
\int_{-\infty}^{x} \lambda \frac{d^{2} u}{d x^{2}} \frac{d u}{d x} d x=\int_{-\infty}^{x} \sum_{i=1}^{N} \sqrt{c_{i} / \lambda}\left(\mu_{i}^{0}-\mu_{i}\right) \frac{d u}{d x} d x
$$

Integrating by parts,

$$
\int_{-\infty}^{x} \frac{d^{2} u}{d x^{2}} \frac{d u}{d x} d x=\left.\left(\frac{d u}{d x}\right)^{2}\right|_{-\infty} ^{x}-\int_{-\infty}^{x} \frac{d u}{d x} \frac{d^{2} u}{d x^{2}} d x,
$$

we reach that at the point $x$

$$
\left(\frac{d u}{d x}(x)\right)^{2}=2 \int_{-\infty}^{x} \frac{d^{2} u}{d x^{2}} \frac{d u}{d x} d x
$$

We now turn to consider the right-hand side of (2.16). From the relation given by (2.15), we find that

$$
\begin{aligned}
\sum_{i=1}^{N} \sqrt{c_{i} / \lambda}\left(\mu_{i}^{0}-\mu_{i}\right) \frac{d u}{d x} & =\left(\sum_{i=1}^{N} \sqrt{c_{i} / \lambda}\left(\mu_{i}^{0}-\mu_{i}\right)\right)\left(\sum_{j=1}^{N} \sqrt{c_{j} / \lambda} \frac{d n_{j}}{d x}\right) \\
& =\frac{1}{\lambda} \sum_{i=1}^{N} \sum_{j=1}^{N} \sqrt{c_{i} c_{j}}\left(\mu_{i}^{0}-\mu_{i}\right) \frac{d n_{j}}{d x} \\
& =\frac{1}{\lambda} \sum_{i=1}^{N} \sum_{j=1}^{N} c_{i}\left(\mu_{j}^{0}-\mu_{j}\right) \frac{d n_{j}}{d x} \\
& =\sum_{j=1}^{N}\left(\mu_{j}^{0}-\mu_{j}\right) \frac{d n_{j}}{d x} .
\end{aligned}
$$


By the definition of the function $\Phi$, the right-hand side of (2.16) is expressed as

$$
\begin{aligned}
\int_{-\infty}^{x} \sum_{i=1}^{N} \sqrt{c_{i} / \lambda}\left(\mu_{i}^{0}-\mu_{i}\right) \frac{d u}{d x} d x & =\int_{-\infty}^{x} \sum_{i=1}^{N}\left(\mu_{i}^{0}-\mu_{i}\right) d n_{i} \\
& =\int_{-\infty}^{x} d \Phi=\Phi(\mathbf{n})-\Phi_{B}
\end{aligned}
$$

It is followed from (2.16), (2.18) and (2.20) that

$$
\lambda\left(\frac{d u}{d x}\right)^{2}=2\left(\Phi(\mathbf{n})-\Phi_{B}\right) .
$$

The grand thermodynamic potential $\Phi$ attains its minimum only when a mixture system is in the equilibrium bulk phases, so the relation $\Phi(\mathbf{n})>\Phi_{B}$ always holds within the two-phase interface region. As the mixture system tends towards to the equilibrium bulk phases, i.e. $x \rightarrow \pm \infty$, we get $\Phi(\mathbf{n}) \rightarrow \Phi_{B}$. These two physical facts are also verified in the numerical tests, and thus, they are assumed to hold in this paper; that is, $\Phi(\mathbf{n})>\Phi_{B}$ for $x \in(-\infty,+\infty)$ and $\Phi(\mathbf{n}) \rightarrow \Phi_{B}$ as $x \rightarrow \pm \infty$.

The monotonicity of $u(x)$ is a direct result of the above physical facts and the equation (2.21). In fact, suppose that the monotonicity of $u(x)$ is not true, then we deduce that there exists one interval in which $\frac{d u}{d x}>0$, and moreover, there exists another interval in which $\frac{d u}{d x}<0$. By the intermediate value theorem, we obtain that there exists at least one point $\widehat{x}$ within the interface such that $\frac{d u}{d x}(\widehat{x})=0$, and as a result, $\Phi(\mathbf{n})=\Phi_{B}$ at $\widehat{x}$. This is in contradiction to the above physical property of the grand thermodynamic potential.

According to the boundary condition given in (1.4), we take the bulk gas phase as the left-hand end, and the right-hand end is the bulk liquid phase. As shown in the numerical results, we assume $u^{G}<u^{L}$, and thus, with the monotonicity of $u(x)$, we claims that $\frac{d u}{d x}>0$ and in this case, $u(x)$ is an increasing function within the two-phase interface region. It is obtained from (2.21) that

$$
\frac{d u}{d x}=\sqrt{\frac{2}{\lambda}\left(\Phi(\mathbf{n})-\Phi_{B}\right)}
$$

By the inverse function rule, the spatial coordinate variable $x$ can be viewed as a function of $u$, and thus, from (2.22), we get

$$
\frac{d x}{d u}=\sqrt{\frac{\lambda}{2\left(\Phi(\mathbf{n})-\Phi_{B}\right)}} .
$$

The boundary values $u^{G}$ and $u^{L}$ can be calculated from the compositions $\mathbf{n}^{G}$ and $\mathbf{n}^{L}$ of the bulk equilibrium phases. So in what follows, the main problem is to design the models and numerical methods for calculating the spatial coordinate variable $x$.

3. Numerical algorithms. The monotonicity of $u$ allows us to develop two efficient methods for calculating the interface, and thus, the boundary problem on the infinite interval can be translated into that in the finite interval $\left[u^{G}, u^{L}\right]$. With the definition of $u$, we can also reduce the expression of the surface tension. From this, we will propose the efficient and accurate methods for computing both the interface and its interior compositions. 
3.1. Computation of the compositions. As shown in subsection 2.3, for a given value of $u \in\left(u^{G}, u^{L}\right)$, there exists a corresponding spatial coordinate $x(u)$ in the interface, and as a result of the existence of the solution $\mathbf{n}$ of the Euler-Lagrange equations, we have the mixture composition at $x(u)$; that is, the mixture composition $\mathbf{n}$ can be viewed as a function of $u$, denoted by $\mathbf{n}(u)$. Based on the Euler-Lagrange equations and the relation (2.3) between $u$ and $\mathbf{n}$, we note that for a given $u$, the corresponding component composition $\mathbf{n}$ can be calculated by solving the following nonlinear system

$$
\begin{gathered}
\sum_{i=1}^{N} \sqrt{c_{i} / \lambda} n_{i}=u, \\
\sqrt{c_{1}}\left(\mu_{i}^{0}(\mathbf{n})-\mu_{i}\right)=\sqrt{c_{i}}\left(\mu_{1}^{0}(\mathbf{n})-\mu_{1}\right), \quad 2 \leq i \leq N .
\end{gathered}
$$

This system can be solved by using Newton's method. For any given approximation $\mathbf{n}^{k}$ of $\mathbf{n}$, the linearized approach is applied for $\delta \mu_{i}$ as

$$
\delta \mu_{i}=\mu_{i}^{0}(\mathbf{n})-\mu_{i} \approx \mu_{i}^{0}\left(\mathbf{n}^{k}\right)-\mu_{i}+\nabla \mu_{i}^{0}\left(\mathbf{n}^{k}\right) \cdot\left(\mathbf{n}-\mathbf{n}^{k}\right), \quad 1 \leq i \leq N .
$$

where $\nabla \mu_{i}^{0}(\mathbf{n})=\left[\frac{\partial \mu_{i}^{0}(\mathbf{n})}{\partial n_{1}}, \cdots, \frac{\partial \mu_{i}^{0}(\mathbf{n})}{\partial n_{N}}\right]^{T}$. A new approximation $\mathbf{n}^{k+1}$ of $\mathbf{n}$ is obtained by solving

$$
\begin{gathered}
\sum_{i=1}^{N} \sqrt{c_{i} / \lambda} n_{i}^{k+1}=u, \\
\left(\sqrt{c_{1}} \nabla \mu_{i}^{0}\left(\mathbf{n}^{k}\right)-\sqrt{c_{i}} \nabla \mu_{1}^{0}\left(\mathbf{n}^{k}\right)\right) \cdot\left(\mathbf{n}^{k+1}-\mathbf{n}^{k}\right) \\
=\sqrt{c_{i}}\left(\mu_{1}^{0}\left(\mathbf{n}^{k}\right)-\mu_{1}\right)-\sqrt{c_{1}}\left(\mu_{i}^{0}\left(\mathbf{n}^{k}\right)-\mu_{i}\right), \quad 2 \leq i \leq N .
\end{gathered}
$$

Let $a_{i, j}(\mathbf{n})=\frac{\partial \mu_{i}^{0}(\mathbf{n})}{\partial n_{j}}=\frac{\partial^{2} f_{0}(\mathbf{n})}{\partial n_{i} \partial n_{j}}$. Define the matrix function $H(\mathbf{n})$ as

$$
H(\mathbf{n})=\left(\begin{array}{ccc}
\sqrt{c_{1} / \lambda} & \cdots & \sqrt{c_{N} / \lambda} \\
\sqrt{c_{1}} a_{2,1}-\sqrt{c_{2}} a_{1,1} & \cdots & \sqrt{c_{1}} a_{2, N}-\sqrt{c_{2}} a_{1, N} \\
\cdots & \cdots & \cdots \\
\sqrt{c_{1}} a_{N, 1}-\sqrt{c_{N}} a_{1,1} & \cdots & \sqrt{c_{1}} a_{N, N}-\sqrt{c_{N}} a_{1, N}
\end{array}\right)
$$

Furthermore, define the vector function as $B(\mathbf{n})=\left[b_{1}(\mathbf{n}), \cdots, b_{N}(\mathbf{n})\right]^{T}$, where

$$
b_{i}(\mathbf{n})=\left(\sqrt{c_{1}} \nabla \mu_{i}^{0}(\mathbf{n})-\sqrt{c_{i}} \nabla \mu_{1}^{0}(\mathbf{n})\right) \cdot \mathbf{n}+\sqrt{c_{i}}\left(\mu_{1}^{0}(\mathbf{n})-\mu_{1}\right)-\sqrt{c_{1}}\left(\mu_{i}^{0}(\mathbf{n})-\mu_{i}\right) .
$$

Denote a special $N$-dimensional vector by $\mathbf{e}_{1}=[1,0, \cdots, 0]^{T}$. For any given $u \in$ $\left(u^{G}, u^{L}\right)$, the corresponding mixture composition $\mathbf{n}$ can be computed by the following iterative method

$$
\mathbf{n}^{k+1}=H\left(\mathbf{n}^{k}\right)^{-1}\left(B\left(\mathbf{n}^{k}\right)+u \mathbf{e}_{1}\right) .
$$


3.2. Direct method for capturing the interface. We now derive the numerical methods for calculating the spatial coordinate variable $x$ based on the equation (2.22). Let $x^{0}$ be the approximate gas-phase end of the two-phase interface. Furthermore, define a finite sequence of different spatial points $x^{i}, i=1, \cdots, M$ and denote $h_{i}=x^{i+1}-x^{i}$. We now consider to solve the equation (2.22), associated with the boundary conditions $u\left(x^{0}\right)=u^{G}$ and $u\left(x^{M}\right)=u^{L}$. Integrating (2.22) over the interval $\left(x^{i}, x^{i+1}\right)$ yields

$$
u\left(x^{i+1}\right)=u\left(x^{i}\right)+\int_{x^{i}}^{x^{i+1}} \sqrt{\frac{2}{\lambda}\left(\Phi(\mathbf{n})-\Phi_{B}\right)} d x .
$$

Let $u^{i}$ be the approximation of $u\left(x^{i}\right)$, and further denote $\mathbf{n}^{i}=\mathbf{n}\left(u^{i}\right)$. We use the $\theta$-scheme [14] for (3.8) to obtain

$$
u^{i+1}=u^{i}+h_{i}\left(\theta \sqrt{\frac{2}{\lambda}\left(\Phi\left(\mathbf{n}^{i}\right)-\Phi_{B}\right)}+(1-\theta) \sqrt{\frac{2}{\lambda}\left(\Phi\left(\mathbf{n}^{i+1}\right)-\Phi_{B}\right)}\right) .
$$

where $\theta \in[0,1]$. A class of methods is obtained from (3.9) as

$$
x^{i+1}=x^{i}+\frac{u^{i+1}-u^{i}}{\theta \sqrt{\frac{2}{\lambda}\left(\Phi\left(\mathbf{n}^{i}\right)-\Phi_{B}\right)}+(1-\theta) \sqrt{\frac{2}{\lambda}\left(\Phi\left(\mathbf{n}^{i+1}\right)-\Phi_{B}\right)}} .
$$

Since $\Phi\left(\mathbf{n}^{G}\right)=\Phi\left(\mathbf{n}^{L}\right)=\Phi_{B}$, we choose $\theta \neq 1$ for $i=0$ and choose $\theta \neq 0$ for $i=M-1$ in $(3.10)$.

3.3. Inverse method for capturing the interface. The two-phase interface region can be determined by solving the equation (2.23). However, the numerical methods for solving (2.23) need to be carefully designed since $\Phi\left(\mathbf{n}\left(u^{G}\right)\right)=\Phi\left(\mathbf{n}\left(u^{L}\right)\right)=$ $\Phi_{B}$.

Divide the interval $\left[u^{G}, u^{L}\right]$ into $M$ sub-intervals $\left[u^{i}, u^{i+1}\right]$, where $i=0,1, \cdots, M-$ 1. Integrating (2.23) over the interval $\left[u^{i}, u^{i+1}\right]$ yields

$$
x\left(u^{i+1}\right)=x\left(u^{i}\right)+\int_{u^{i}}^{u^{i+1}} \sqrt{\frac{\lambda}{2\left(\Phi(\mathbf{n}(u))-\Phi_{B}\right)}} d u .
$$

Denote by $x^{i}$ the approximation of $x\left(u^{i}\right)$. For $i=1, \cdots, M-2$, the $\theta$-scheme used for (3.11) gives us

$$
x^{i+1}=x^{i}+\left(u^{i+1}-u^{i}\right)\left(\theta \sqrt{\frac{\lambda}{2\left(\Phi\left(\mathbf{n}^{i}\right)-\Phi_{B}\right)}}+(1-\theta) \sqrt{\frac{\lambda}{2\left(\Phi\left(\mathbf{n}^{i+1}\right)-\Phi_{B}\right)}}\right) 3
$$

where $\theta \in[0,1]$. Since $\Phi\left(\mathbf{n}^{G}\right)=\Phi\left(\mathbf{n}^{L}\right)=\Phi_{B}$, we choose $\theta=0$ for $i=0$ and choose $\theta=1$ for $i=M-1$ in (3.12). The initial point $x^{0}$ is chosen as the approximation of $x\left(u^{G}\right)$.

3.4. Computation of the surface tension. Firstly, we note that the expression of surface tension can be reduced as

$$
\sigma=\int_{-\infty}^{+\infty}\left(\Phi(\mathbf{n})-\Phi_{B}+\frac{1}{2} \lambda\left(\frac{d u}{d x}\right)^{2}\right) d x
$$




$$
\begin{aligned}
& =\int_{-\infty}^{+\infty} \lambda\left(\frac{d u}{d x}\right)^{2} d x \\
& =\int_{-\infty}^{+\infty} 2\left(\Phi(\mathbf{n})-\Phi_{B}\right) d x .
\end{aligned}
$$

We have shown in subsection 3.1 that for given $u$, the corresponding composition $\mathbf{n}$ can be computed by solving (3.1) and (3.2), it is found from (2.23) and (3.13) that the surface tension can be expressed as

$$
\sigma=\int_{u^{G}}^{u^{L}} \sqrt{2 \lambda\left(\Phi(\mathbf{n}(u))-\Phi_{B}\right)} d u .
$$

Using the trapezoid rule to the right-hand side of (3.14), we obtain the approximation of the surface tension as

$$
\sigma \simeq \sum_{i=0}^{M-1} \frac{1}{2}\left(u^{i+1}-u^{i}\right)\left(\sqrt{2 \lambda\left(\Phi\left(\mathbf{n}^{i}\right)-\Phi_{B}\right)}+\sqrt{2 \lambda\left(\Phi\left(\mathbf{n}^{i+1}\right)-\Phi_{B}\right)}\right) .
$$

3.5. A summary description of numerical algorithms. As shown above, we have developed two methods to capture the interface between two phases, and combining these two methods with the computation of the surface tension, we propose the following algorithms:

(1) Calculate the components $\mathbf{n}^{G}$ and $\mathbf{n}^{L}$ in the equilibrium bulk phases by PengRobinson equation of state, and then compute $u^{G}$ and $u^{L}$;

(2) Divide the interval $\left[u^{G}, u^{L}\right]$ into $M$ subintervals $\left[u^{i}, u^{i+1}\right]$, where $i=0, \cdots, M-1$, and $u^{0}=u^{G}, u^{M}=u^{L}$, and then compute the corresponding components $\mathbf{n}^{i}=\mathbf{n}\left(u^{i}\right)$ by the iterative method (3.7);

(3) Calculate the approximate surface tension by (3.15) and calculate the spatial points $x^{i}$ by the proposed direct method or the proposed inverse method.

The main advantage of the proposed methods is that no partial differential equation is needed to be solved, and compared to the known similar method, not only the proposed methods are supported by the rigorous theoretical foundation shown in this paper, but also they do not need to choose any reference component. In the well known method of reference component, one needs to choose a reference component by predicting rough profiles of component molar densities before simulation.

4. Numerical tests. We now simulate the surface tension by using the proposed methods for three hydrocarbon mixtures composed of multiple components. The Peng-Robinson equation of state [24] is used to compute the equilibrium properties of the bulk phases, such as the densities and the chemical potentials. In all tests, a uniform mesh with 500 elements is applied for the weighted molar density domain $\left[u^{G}, u^{L}\right]$, and we take the parameter $\theta=1 / 2$ for both methods given by (3.10) and (3.12).

4.1. Binary mixture. We simulate the surface tension of a binary mixture, which is composed of methane $\left(\mathrm{CH}_{4}\right)$ and decane $\left(\mathrm{nC}_{10}\right)$.

Surface tensions of the mixture computed by the two methods at $310 \mathrm{~K}$ and different pressures are illustrated in Figure 4.1. We observe the drop of the calculated the surface tension as the pressures increase within a certain range, which are in agreement with the ones presented in [20]. 

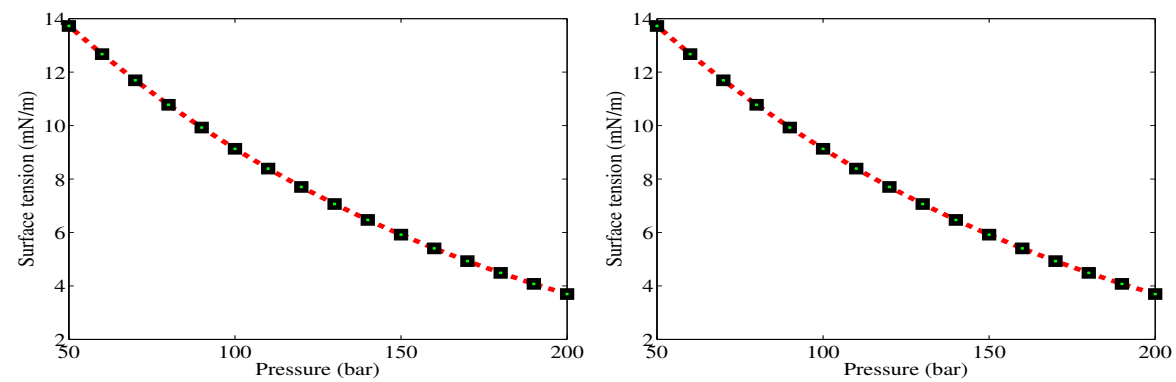

Fig. 4.1: Surface tension of $\mathrm{CH}_{4}$ and $\mathrm{nC}_{10}$ mixture computed by two methods at 310K: Direct method (left), Inverse method (right)

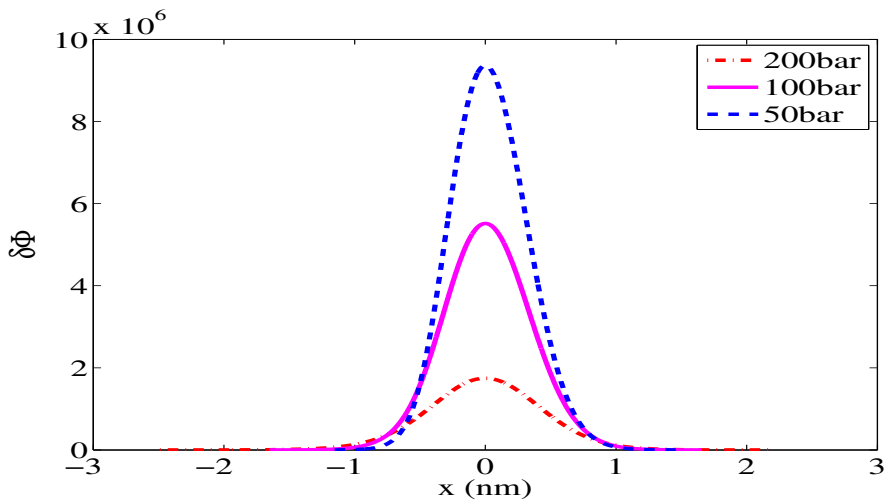

Fig. 4.2: The graphics of function $\delta \Phi$ for the binary mixture

The graphics of function $\Phi(\mathbf{n}(u))-\Phi_{B}$, denoted by $\delta \Phi$, are drawn in Figure 4.2 at different pressures. It can be observed that $\delta \Phi$ are larger than zero except for the bulk phase ends, and $\delta \Phi$ attains its maximum at about the middle of the interface region.

Figures 4.3 illustrate the density profiles calculated by the direct method at $310 \mathrm{~K}$ and different pressures. From these figures, we can observe that the proposed methods not only accurately compute the surface tension of the tested binary mixture, but also efficiently capture the interfaces and calculate their interior compositions.

Figures 4.4 depict the calculated weighted molar density $(u)$ profiles at $310 \mathrm{~K}$ and different pressures. It is shown that the weighted molar density of the tested binary mixture is always increasing monotonically across the interface thickness at different pressures. This verifies the theoretical result about the monotonicity property of $u$.

It can be seen that the methane molar densities do not vary monotonically with the spatial coordinate and their maximum values in the interface increase with pressures. Although the decane molar density is monotonically increasing, the weighted molar density differences between gas and liquid phases are all less than those of the decane; that is, the weighted molar densities vary slower than any component molar density. This means that the method of using the weighted molar density may be more stable than the selection of the reference component.

4.2. Ternary mixture. We calculate the surface tension of a ternary mixture, which is composed of $75 \%$ methane $\left(\mathrm{CH}_{4}\right), 15 \%$ pentane $\left(\mathrm{C}_{5}\right)$ and $10 \%$ decane $\left(\mathrm{nC}_{10}\right)$ at the temperature $313 \mathrm{~K}$. 

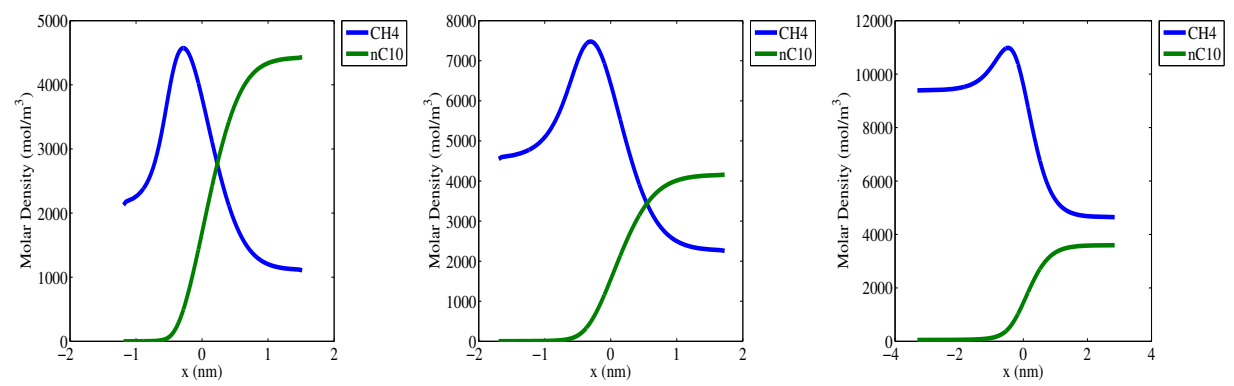

Fig. 4.3: $\mathrm{CH}_{4}$ and $\mathrm{nC}_{10}$ molar density profiles computed by the direct method at 310K and different pressures: 50bar (left), 100bar (center), 200bar (right)
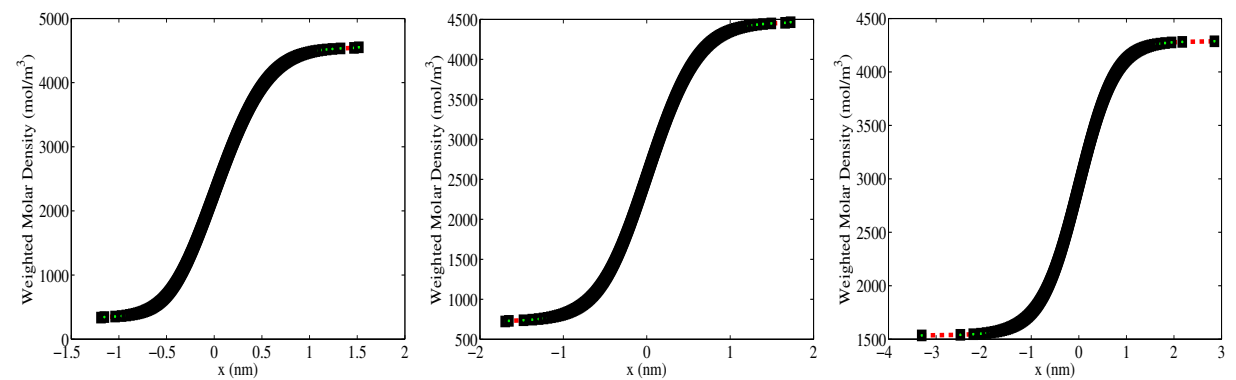

Fig. 4.4: $\mathrm{CH}_{4}$ and $\mathrm{nC}_{10}$ weighted molar density profiles computed by the direct method at 310K and different pressures: 50bar (left), 100bar (center), 200bar (right)

Surface tensions of the mixture computed by the two methods at $313 \mathrm{~K}$ and different pressures are illustrated in Figure 4.5. The graphics of function $\delta \Phi(u)$ are drawn in Figure 4.6 at different pressures. Figures 4.7 depict the component molar density profiles calculated by the direct method at $313 \mathrm{~K}$ and different pressures. Figures 4.8 show the calculated weighted molar density profiles at $313 \mathrm{~K}$ and different pressures, which also verify the monotonicity of $u$ in the space.

4.3. Five-component mixture. The hydrocarbon mixture is composed of $80 \%$ of methane $\left(\mathrm{CH}_{4}\right), 14 \%$ n-butane $\left(\mathrm{nC}_{4}\right), 4 \%$ n-heptane $\left(\mathrm{nC}_{7}\right), 1.4 \%$ decane $\left(\mathrm{nC}_{10}\right)$ and $0.6 \%$ n-tetradecane $\left(\mathrm{nC}_{14}\right)$.

Figures 4.9 illustrate the surface tensions of the mixture computed by the two methods at $313 \mathrm{~K}$ and different pressures. The graphics of function $\delta \Phi(u)$ are drawn in Figure 4.10 at different pressures. Figures 4.11 show the component molar density profiles calculated by the direct method at $313 \mathrm{~K}$ and different pressures. Figures 4.12 illustrate the calculated weighted molar density profiles at $313 \mathrm{~K}$ and different pressures.

From the last two examples, we also observe that the proposed methods not only accurately compute the surface tension, but also efficiently capture the interfaces and calculate their interior compositions. We also get the similar analytic conclusions to those presented in subsection 4.1. These numerical results demonstrate the proposed method is an ideal instrument to simulate the surface tension problems.

Finally, we must note that the numerical results of molar densities and weighted molar densities computed by the inverse method are very similar to those of the direct method, so we omit their illustrations for sake of simplicity. 

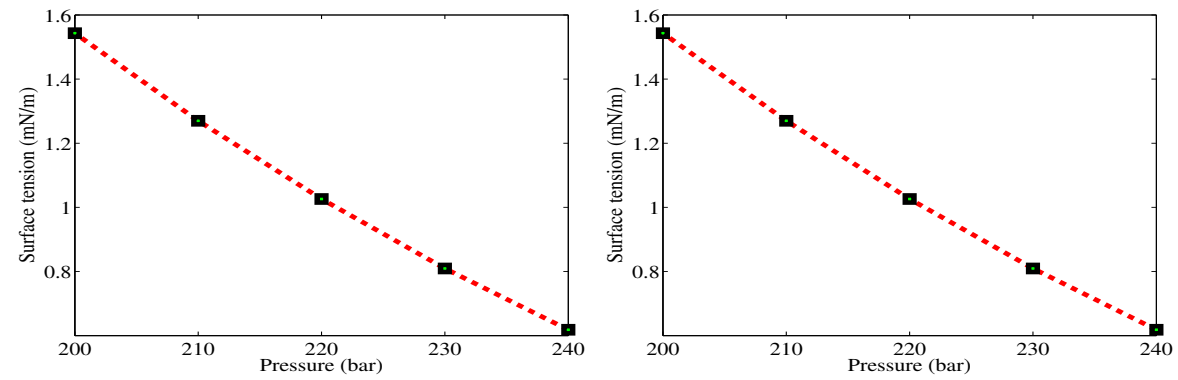

Fig. 4.5: Surface tension of $\mathrm{CH}_{4}, \mathrm{C}_{5}$ and $\mathrm{nC}_{10}$ mixture computed by two methods at 313K: Direct method (left), Inverse method (right)

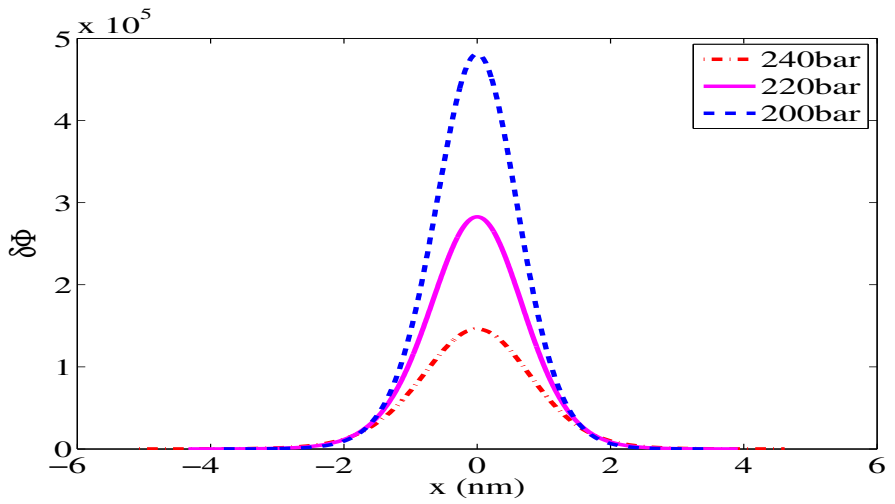

Fig. 4.6: The graphics of function $\delta \Phi$ for the ternary mixture
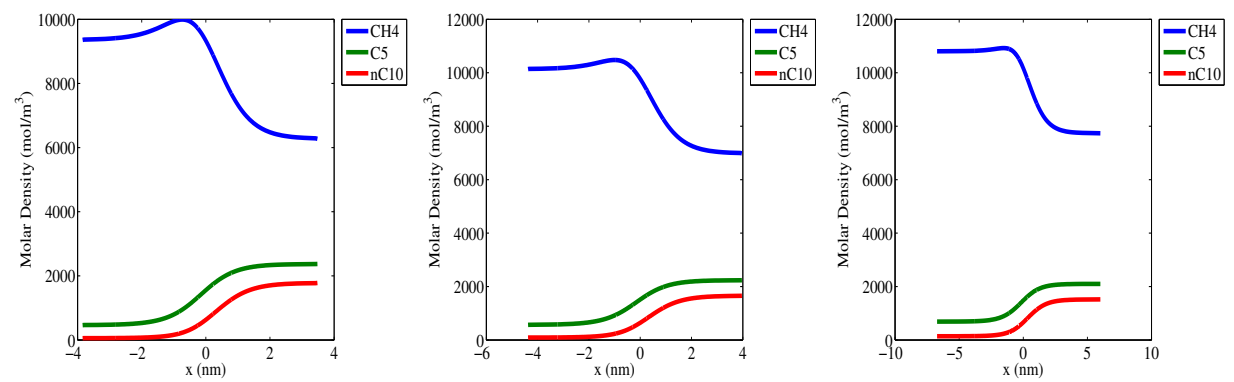

Fig. 4.7: $\mathrm{CH}_{4}, \mathrm{C}_{5}$ and $\mathrm{nC}_{10}$ molar density profiles computed by the direct method at $313 \mathrm{~K}$ and different pressures: 200bar (left), 220bar (center), 240bar (right)
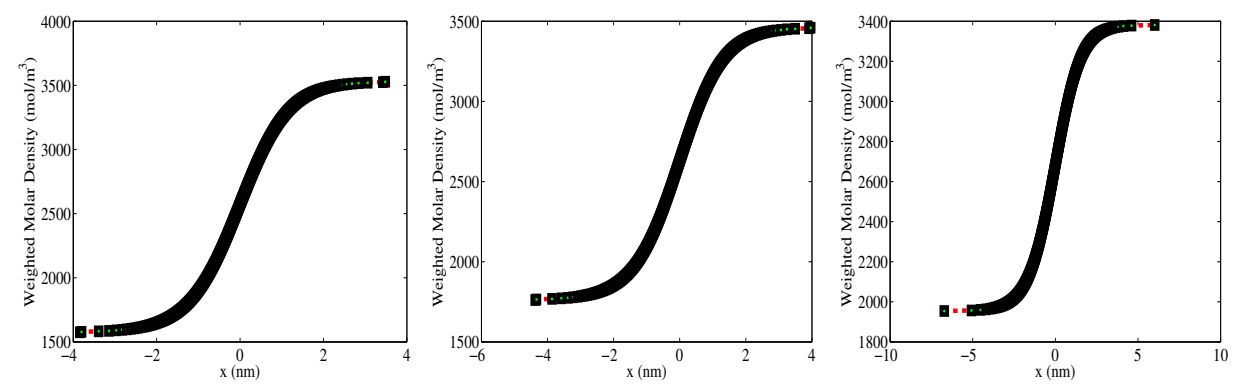

Fig. 4.8: $\mathrm{CH}_{4}, \mathrm{C}_{5}$ and $\mathrm{nC}_{10}$ weighted molar density profiles computed by the direct method at 313K and different pressures: 200bar (left), 220bar (center), 240bar (right) 

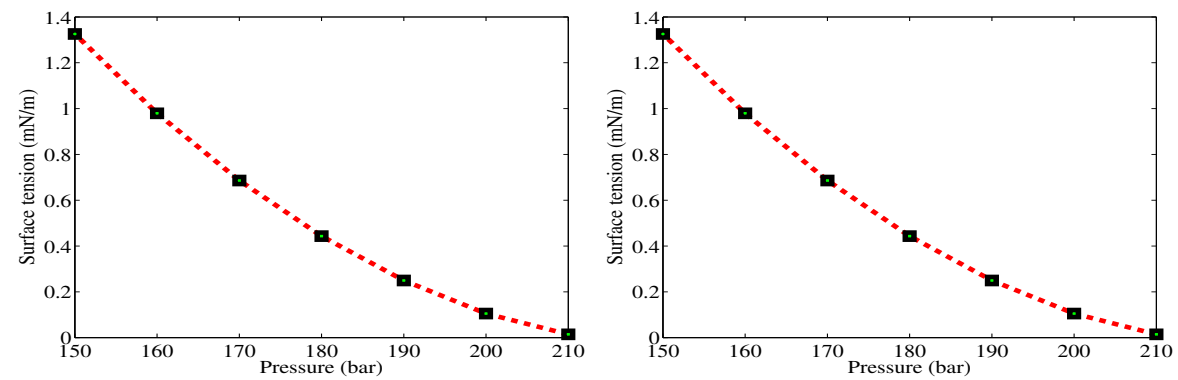

Fig. 4.9: Surface tension of five-component mixture computed by two methods at 313K: Direct method (left), Inverse method (right)

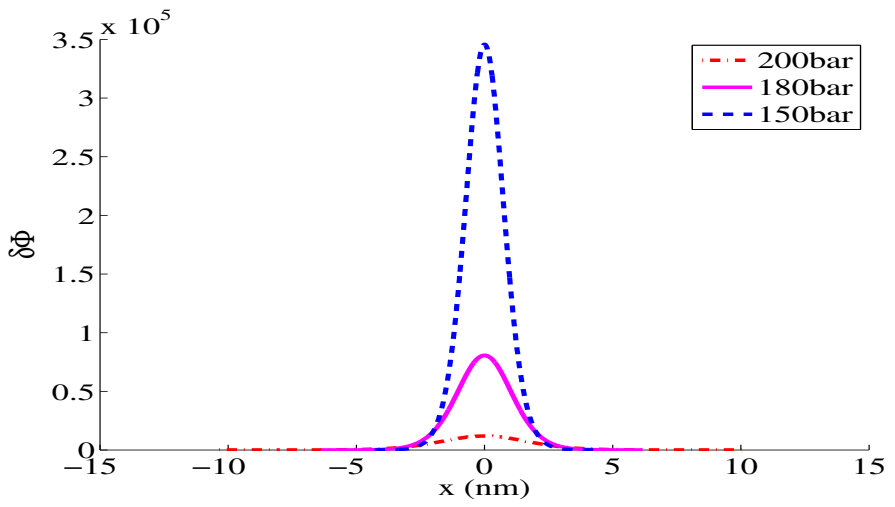

Fig. 4.10: The graphics of function $\delta \Phi$ for the five-component mixture
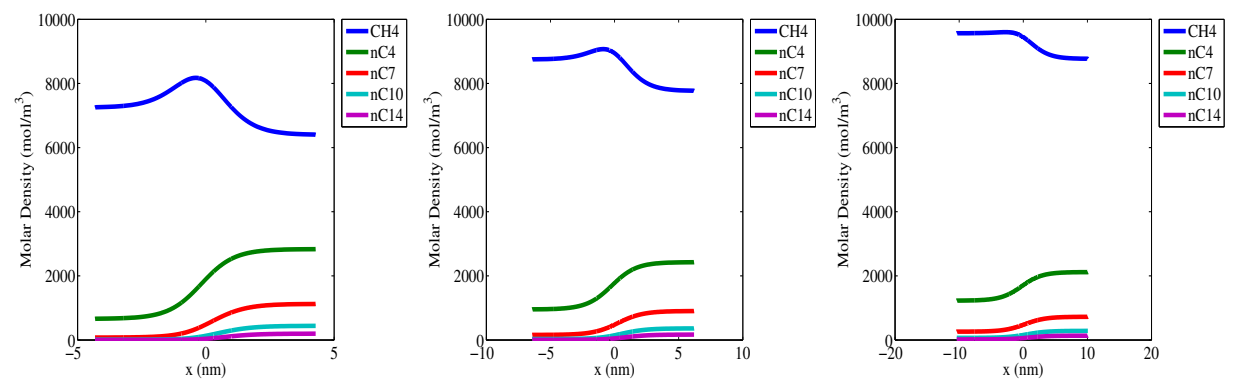

Fig. 4.11: Five-component molar density profiles computed by the direct method at 313K and different pressures: 150bar (left), 180bar (center), 200bar (right)
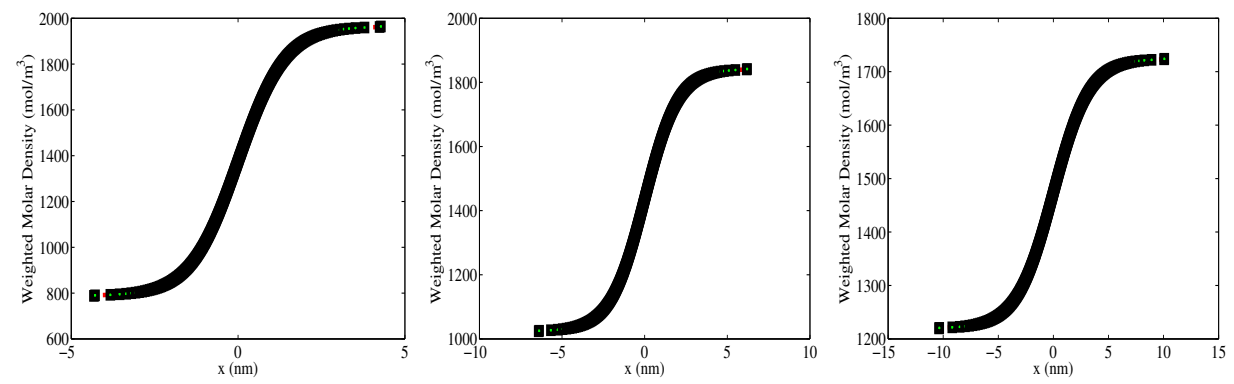

Fig. 4.12: Five-component weighted molar density profiles computed by the direct method at 313K and different pressures: 150bar (left), 180bar (center), 200bar (right) 
5. Conclusions. A well-defined path function has been constructed based on a linear transformation, and then the expressions of the Euler-Lagrange equations are reduced. Furthermore, we have proven the monotonicity of the path function, and from this, we develop two efficient methods for calculating the two-phase fluid interface and computing the surface tension. Compared to the known methods, the proposed methods not only eliminate the need of solving a differential equation system, but also are easier to be implemented in practical applications. The numerical examples are also given to verify the proposed theory and demonstrate the efficiency of the proposed methods.

\section{Appendix A. Computations of $f_{0}$.}

The Helmholtz energy $f_{0}(\mathbf{n})$ of a homogeneous fluid is given by

$$
f_{0}(\mathbf{n})=f_{0}^{\text {ideal }}(\mathbf{n})+f_{0}^{\text {excess }}(\mathbf{n}) .
$$

The ideal gas contribution is

$$
f_{0}^{\text {ideal }}(\mathbf{n})=R T \sum_{i=1}^{N} n_{i}\left(\ln n_{i}-1\right) .
$$

The excess (non-ideal) contribution, for Peng-Robinson fluids, is

$$
f_{0}^{\text {excess }}(\mathbf{n})=-n R T \ln (1-b n)+\frac{a(T) n}{2 \sqrt{2} b} \ln \left(\frac{1+(1-\sqrt{2}) b n}{1+(1+\sqrt{2}) b n}\right),
$$

where $T$ is the temperature of the mixture and $R$ is the universal gas constant. Here, $b$ is the covolume and $a(T)$ is the energy parameter.

The parameters $a(T)$ and $b$ in (A.3) are computed as below. Let $P$ be the pressure of the mixture and let $T_{c_{i}}$ and $P_{c_{i}}$ be critical temperature and critical pressure, respectively, of component $i$. For the $i$ th component, we define the reduced pressure as $P_{r_{i}}=P / P_{c_{i}}$ and the reduced temperature as $T_{r_{i}}=T / T_{c_{i}}$, and furthermore, we define the mole fraction $z_{i}=n_{i} / n$, where $n=\sum_{i=1}^{N} n_{i}$. Then $a(T)$ and $b$ are calculated by

$$
a(T)=\sum_{i=1}^{N} \sum_{j=1}^{N} z_{i} z_{j}\left(a_{i} a_{j}\right)^{1 / 2}\left(1-k_{i j}\right), \quad b=\sum_{i=1}^{N} z_{i} b_{i},
$$

where $k_{i j}$ is the given binary interaction coefficients for the energy parameters and

$$
a_{i}=0.45724 \frac{R^{2} T_{c_{i}}^{2}}{P_{c_{i}}}\left[1+m_{i}\left(1-\sqrt{T_{r_{i}}}\right)\right]^{2}, \quad b_{i}=0.07780 \frac{R T_{c_{i}}}{P_{c_{i}}} .
$$

The coefficients $m_{i}$ are calculated by the following formulations

$$
\begin{gathered}
m_{i}=0.37464+1.54226 \omega_{i}-0.26992 \omega_{i}^{2}, \quad \omega_{i} \leq 0.49, \\
m_{i}=0.379642+1.485030 \omega_{i}-0.164423 \omega_{i}^{2}+0.016666 \omega_{i}^{3}, \quad \omega_{i}>0.49,
\end{gathered}
$$

where $\omega_{i}$ is the acentric factor.

Denote $\mathbf{N}=\left[N_{1}, \cdots, N_{N}\right]^{T}$. We note that (1.2) is equivalent to the following more standard definition of chemical potential:

$$
\mu_{i}^{0}=\left(\frac{\partial F_{0}(\mathbf{N}, T, V)}{\partial N_{i}}\right)_{T, V, N_{1}, \cdots, N_{i-1}, N_{i+1}, \cdots, N_{N}},
$$


which can be proved by recalling the definition of $F_{0}=f_{0} V$ and applying some calculus manipulation.

Appendix B. Influence parameters. The pure component influence parameters $c_{i}$ are given by

$$
c_{i}=a_{i} b_{i}^{2 / 3}\left[\alpha_{i}\left(1-T_{r_{i}}\right)+\beta_{i}\right],
$$

where $\alpha_{i}$ and $\beta_{i}$ are the coefficients correlated merely with the acentric factor $\omega_{i}$ of the component $i$ by the following relations

$$
\alpha_{i}=-\frac{10^{-16}}{1.2326+1.3757 \omega_{i}}, \quad \beta_{i}=\frac{10^{-16}}{0.9051+1.5410 \omega_{i}} .
$$

\section{REFERENCES}

[1] D. M. Anderson, G. B. McFadden, and A. A. Wheeler. Diffuse-interface methods in fluid mechanics. Annual review of fluid mechanics, 30(1):139-165, 1998.

[2] P. Boyanovaa and M. Neytcheva. Efficient numerical solution of discrete multi-component Cahn-Hilliard systems. Computers and Mathematics with Applications, 67:106-121, 2014.

[3] B. Breure and C. J. Peters. Modeling of the surface tension of pure components and mixtures using the density gradient theory combined with a theoretically derived influence parameter correlation. Fluid Phase Equilibria, 334: 189-196, 2012.

[4] Michael J. Borden, Thomas J.R. Hughes, Chad M. Landis, Clemens V. Verhoosel. A higherorder phase-field model for brittle fracture: Formulation and analysis within the isogeometric analysis framework. Computer Methods in Applied Mechanics and Engineering, 273: 100-118, 2014.

[5] B. S. Carey, L. E. Scriven, and H. T. Davis. Semiempirical theory of surface tension of binary systems. AIChE Journal, 26(5):705-711, 1980.

[6] Feng Chen and Jie Shen. Efficient energy stable schemes with spectral discretization in space for anisotropic Cahn-Hilliard systems. Commun. Comput. Phys., 13:1189-1208, 2013.

[7] P. M. W. Cornelisse. The Square Gradient Theory Applied - Simultaneous Modelling of Interfacial Tension and Phase Behaviour. PhD thesis, TU Delft, The Netherlands, 1997.

[8] Howard Ted Davis. Statistical mechanics of phases, interfaces, and thin films. VCH New York, 1996.

[9] Knut Erik Teigen, Peng Song, John Lowengrub, and Axel Voigt. A diffuse-interface method for two-phase flows with soluble surfactants. Journal of Computational Physics, 230(2):375393, 2011.

[10] A. Firoozabadi. Thermodynamics of hydrocarbon reservoirs. McGraw-Hill, New York, 1999.

[11] H. Gomez, X. Nogueira. An unconditionally energy-stable method for the phase field crystal equation. Computer Methods in Applied Mechanics and Engineering, 249-252: 52-61, 2012.

[12] Junseok Kim. A generalized continuous surface tension force formulation for phase-field models for multi-component immiscible fluid flows. Computer Methods in Applied Mechanics and Engineering, 198(37): 3105-3112, 2009.

[13] Junseok Kim. Phase-field models for multi-component fluid flows. Communications in Computational Physics, 12(3):613-661, 2012.

[14] Petr Kloucek and Franz S. Rys. Stability of the fractional step $\theta$-scheme for the nonstationary Navier-Stokes equations. SIAM Journal on Numerical Analysis, 31(5):1312-1335, 1994.

[15] Jisheng Kou and Shuyu Sun. An adaptive finite element method for simulating surface tension with the gradient theory of fluid interfaces. Journal of Computational and Applied Mathematics, 255:593-604, 2014.

[16] L. W. Lake. Enhanced oil recovery. Prentice Hall, Englewood Cliffs, New Jersey, 1989.

[17] Zhibao Li and Benjamin C.-Y. Lu. On the prediction of surface tension for multicomponent mixtures. The Canadian Journal of Chemical Engineering, 79(3):402-411, 2001. 
[18] Christian Miehe, Gautam Ethiraj. A geometrically consistent incremental variational formulation for phase field models in micromagnetics. Computer Methods in Applied Mechanics and Engineering, 245-246: 331-347, 2012.

[19] Christelle Miqueu, Bruno Mendiboure, Alain Graciaa, and Jean Lachaise. Modelling of the surface tension of pure components with the gradient theory of fluid interfaces: a simple and accurate expression for the influence parameters. Fluid phase equilibria, 207(1):225246, 2003.

[20] Christelle Miqueu, Bruno Mendiboure, Alain Graciaa, and Jean Lachaise. Modeling of the surface tension of multicomponent mixtures with the gradient theory of fluid interfaces. Industrial and Engineering Chemistry Research, 44(9):3321-3329, 2005.

[21] J. Moortgat, S. Sun, and A. Firoozabadi. Compositional modeling of three-phase flow with gravity using higher-order finite element methods. Water Resources Research, 47:W05511, 2011.

[22] Livia A Moreira and Abbas Firoozabadi. Molecular thermodynamic modeling of droplet-type microemulsions. Langmuir, 28(3):1738-1752, 2012.

[23] Britta Nestler and Abhik Choudhury. Phase-field modeling of multi-component systems. Current Opinion in Solid State and Materials Science, 15(3):93-105, 2011.

[24] Ding-Yu Peng and Donald B. Robinson. A new two-constant equation of state. Industrial and Engineering Chemistry Fundamentals, 15(1):59-64, 1976.

[25] Zhonghua Qiao, Zhengru Zhang, and Tao Tang. An adaptive time-stepping strategy for the molecular beam epitaxy models. SIAM Journal on Scientific Computing, 33(3):1395-1414, 2011.

[26] Muhammad Sahimi, H. Davis, and L.E. Scriven. Thermodynamic modeling of phase and tension behavior of co2/hydrocarbon systems. Old SPE Journal, 25(2):235-254, 1985.

[27] S. Sun and J. Liu. A locally conservative finite element method based on enrichment of the continuous Galerkin method. SIAM Journal on Scientific Computing, 31(4):2528-2548, 2009 .

[28] S. Sun and M. F. Wheeler. Analysis of discontinuous Galerkin methods for multicomponent reactive transport problems. Computers and Mathematics with Applications, 52(5):637650, 2006.

[29] S. Sun and M. F. Wheeler. A dynamic, adaptive, locally conservative and nonconforming solution strategy for transport phenomena in chemical engineering. Chemical Engineering Communications, 193(12):1527-1545, 2006.

[30] S. Sun and M. F. Wheeler. Discontinuous Galerkin methods for simulating bioreactive transport of viruses in porous media. Advances in Water Resources, 30(6-7):1696-1710, 2007.

[31] M.F. Wheeler, T. Wick, W. Wollner. An augmented-Lagrangian method for the phase-field approach for pressurized fractures. Computer Methods in Applied Mechanics and Engineering, 271: 69-85, 2014.

[32] Xiongce Zhao, J. Karl Johnson, and Craig E Rasmussen. Surface tension of quantum fluids from molecular simulations. Journal of Chemical Physics, 120(18):8707-8715, 2004. 